RESEARCH PAPER RP1363

Part of Journal of Research of the National Bureau of Standards, Volume 26, February 1941

\title{
OBLIQUE-INCIDENCE RADIO TRANSMISSION AND THE LORENTZ POLARIZATION TERM
}

By Newbern Smith

\begin{abstract}
The force on an electron in an ionized medium may be written $\mathscr{E}+4 \pi a P$ per unit charge, where $\mathscr{E}$ is the electric force, $P$ the polarization of the medium, and $a$ a constant which is zero on the Sellmeyer theory and may have a value of $1 / 3$ on the Lorentz theory. A well-defined distinction exists between maximum usable frequencies for ionospheric radio transmission calculated on the basis of the two theories. This paper describes a type of experiment for measuring maximum usable frequencies and comparing them with calculated values. The results indicate that $a$ is probably zero, at least in these experiments. There is also described a step-by-step method of solving the virtual-height integral equation, necessary in much theoretical work concerning the ionosphere.
\end{abstract}

\section{CONTENTS}

Page

I. Introduction

II. Theory, neglecting the earth's magnetic field $\ldots \ldots \ldots \ldots \ldots$

III. Effect of the earth's magnetic field

IV. Experimental observations_._.

V. Conclusions

VI. References

\section{INTRODUCTION}

The question whether the force on an electron in an ionized medium should be taken as merely the applied electric force, $\mathscr{E}$, or the so-called Lorentz force, $\mathscr{E}+4 \pi a P[1],{ }^{1}$ where $P$ is the polarization of the medium and $a$ is a constant which is usually taken as $1 / 3$, is of fundamental importance in the theory of the ionosphere. The two theories are known, respectively, as the Sellmeyer theory and the Lorentz theory, and the difference between them represents a difference of 50 percent in the equivalent electron density in the ionosphere.

The argument had apparently been settled in favor of the Sellmeyer theory in a comprehensive theoretical paper by Darwin [2], when the whole question was reopened by a radio experiment of Booker and Berkner [3], which they were able to interpret only in terms of the Lorentz theory. In Australia, Martyn and Munro [4] explained the same kind of radio experiment in terms of the Sellmeyer theory, but Appleton, Farmer, and Ratcliffe [5] in England attacked this interpretation.

The radio experiments referred to were all performed at vertical incidence, in the neighborhood of the gyrofrequency, or precessional frequency of electrons in the earth's magnetic field (about $1.4 \mathrm{Mc} / \mathrm{s}$ ). The purpose of this paper is to present the results of a different kind

\footnotetext{
1 Figures in brackets indicate the literature references at the end of this paper.
} 
of radio experiment-one involving ionospheric transmission of radio waves over a distance at oblique incidence, at a high frequency, and during the daylight hours.

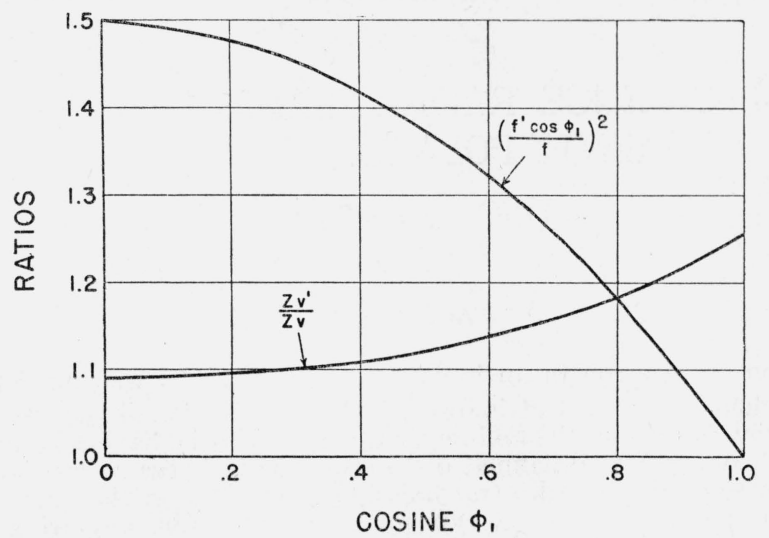

Figure 1.-Oblique-incidence relations for Sellmeyer and Lorentz theories.

$z_{0}^{\prime}=$ height of equivalent triangular path, $a=1 / 3$

$z_{0}=$ height of equivalent triangular path, $a=0$ (equal to virtual height at equivalent vertical-incidence frequency)

$f=$ equivalent vertical-incidence frequency, $a=1 / 3$

$f^{\prime} \cos \phi_{1}=$ equivalent vertical-incidence frequency, $a=0$

$\phi_{1}=$ angle of incidence of waves upon the ionized layers.

\section{THEORY, NEGLECTING THE EARTH'S MAGNETIC FIELD}

There exists a well-defined distinction in the behavior of obliqueincidence radio transmission according to the two theories. This distinction is that the maximum usable frequency for radio sky-wave transmission over a given distance is greater for the Lorentz theory than for the Sellmeyer theory.

In two previous papers $[6,7]$ the author outlined a method of calculating maximum usable frequencies and heights of reflection for oblique-incidence radio transmission directly from the curve of frequency versus virtual height obtained at vertical incidence. This curve will hereafter be called the $\left(z_{0}, f\right)$ curve, where $z_{0}$ is the virtual height and $f$ is the vertical-incidence frequency. The virtual height is obtained by measuring the time, $\Delta t$, taken by a pulse to travel up to the ionosphere and back. It is given by

$$
z_{v}=\frac{c \Delta t}{2}=\int_{0}^{z_{0}} \frac{d}{d f}(\mu f) d z
$$

where $c=$ velocity of light,

$f=$ frequency of waves,

$\mu=$ refractive index of the ionosphere,

$z_{0}=$ actual level of reflection, and

$z=$ measured vertically upward. 
The method of calculating maximum usable frequencies was based on the simple ray theory of a stratified isotropic ionosphere, whose refractive index, $\mu^{\prime}$, for waves of frequency $f^{\prime}$ was given by

$$
\mu^{\prime}=\sqrt{1-\frac{f_{0}^{2}}{f^{\prime 2}}}
$$

where $f^{\prime}$ is the actual wave frequency and $f_{0}^{2}=N e^{2} / \pi m, N$ being the ionization density expressed as a function of height.

The calculation of maximum usable frequencies consists essentially in solving graphically the two equations $z_{v}=z_{v}(f)$, the $\left(z_{v}, f\right)$ curve for vertical incidence, and $z_{v}=z_{0}\left(f, f^{\prime}, D\right)$, the transmission equation for oblique incidence, obtained below. Here $D$ is the distance of transmission, $f^{\prime}$ the wave frequency, and $f$ the equivalent vertical-incidence frequency, or frequency of the wave reflected, at vertical incidence, at the same level as is the wave frequency, $f^{\prime}$, over the given distance, $D$.

The transmission equation is found by combining the expression for the equivalent triangular path, obtained geometrically, with the expression for the equivalent vertical-incidence frequency, $f$, and the relation between $z_{v}$ and $z_{0}{ }^{\prime}$, the height of the equivalent triangular path.

The intersection of the graphs of the two equations gives both the equivalent vertical-incidence frequency, $f$, and the virtual height of reflection corresponding to transmission of the given wave frequency, $f^{\prime}$, over the given distance, $D$. The greatest value of $f^{\prime}$ for which a real solution exists is the maximum usable frequency over the given distance.

When the Lorentz polarization term $a$ is included, the refractive index, $\mu^{\prime}$, is given [8] by

$$
\mu^{\prime}=\sqrt{1-\frac{f_{0}{ }^{2}}{f^{\prime 2}+a f_{0}{ }^{2}}} .
$$

The virtual height at the vertical-incidence frequency, $f$, is

$$
z_{v}=\int_{0}^{z_{0}} \frac{1-a\left(1-\mu^{2}\right)^{2}}{\mu} d z,
$$

where $z_{0}$ is the level of reflection, and $\mu$ is $\mu^{\prime}$ with $f$ substituted for $f^{\prime}$. The equivalent vertical-incidence frequency, $f$, is given by

$$
f=f^{\prime} \cos \phi_{1} \sqrt{\frac{1-a}{1-a \cos ^{2} \phi_{1}}},
$$

where $\phi_{1}$ is the angle of incidence of the actual wave upon the ionosphere.

The height, $z_{v}{ }^{\prime}$, of the equivalent triangular path may be calculated as follows. Let $\phi$ be the angle between the ray path and the vertical at the height $z$. Then 


$$
z_{v}^{\prime}=\cos \phi_{1} \int_{0}^{z_{0}} \frac{d z}{\mu^{\prime} \cos \phi}=\cos \phi_{1} \int_{0}^{z_{0}} \frac{d z}{\sqrt{\cos ^{2} \phi_{1}-\frac{f_{0}^{2}}{f^{\prime 2}+a f_{0}^{2}}}} .
$$

This becomes, on substituting $f$ for $f^{\prime}$,

$$
z_{v}^{\prime}=\int_{0}^{z_{0}} \sqrt{\frac{f^{2}+a \frac{(1-a) \cos ^{2} \phi_{1}}{1-a \cos ^{2} \phi_{1}} f_{0}^{2}}{f^{2}-(1-a) f_{0}^{2}}} d z .
$$

To obtain the transmission equation, we get geometrically the value of

$$
z_{v}^{\prime}=z_{v}^{\prime}\left(D, \phi_{1}\right) \text {. }
$$

We then insert in eq 6 the value of $\phi_{1}$ obtained from eq 4 in terms of $f$ and $f^{\prime}$, and multiply $z_{0}^{\prime}$ by the ratio $z_{0}: z_{0}^{\prime}$ determined by comparing eq 3 and 5 .

This analysis has been made for a flat ionosphere, but may be extended to a curved ionosphere in the manner indicated in reference [7].

For $a=1 / 3$, the ratio $f^{\prime} / f$ is greater than for $a=0$, and so the wave frequency, $f^{\prime}$, corresponding to a given $f$ and $z_{v}$ is in general greater. If $z_{v}$ were equal to $z_{v}{ }^{\prime}$, therefore, the calculated maximum usable frequency would be much greater for $a=1 / 3$ than for $a=0$, the difference increasing with the angle of incidence, $\phi_{1}$. We must, however, examine the relation between $z_{v}$ and $z_{v}{ }^{\prime}$. Now $z$ is a function of $f_{0}{ }^{\prime}$, so if we put $x \equiv f_{0}^{2} / f^{2}$ we may rewrite eq 3 and 5 :

$$
\begin{gathered}
z_{v}=\int_{0}^{\frac{1}{1-a}} \frac{1-a \frac{x^{2}}{(1+a x)^{2}}}{\sqrt{1-\frac{x}{1+a x}}} \frac{d z}{d x} d x . \\
z_{v}^{\prime}=\int_{0}^{\frac{1}{1-a}} \sqrt{\frac{1+a \frac{(1-a) \cos ^{2} \phi_{1}}{1-a \cos ^{2} \phi_{1}} x}{1-(1-a) x}} \frac{d z}{d x} d x .
\end{gathered}
$$

Equation 7 gives $z_{v}$ as a function of $f^{2}$ and eq 8 gives $z_{v}{ }^{\prime}$ as a function of $f^{2}$ and $\cos \phi_{1}$, so that $z_{v} / z_{v}{ }^{\prime}$ may be expressed as a function of $f^{2}$ and $\cos \phi_{1}$.

The ratio $z_{v} / z_{0}^{\prime}$ is, for $a=1 / 3$, in general less than unity, that is, less than the value it had for $a=0$. This tends to decrease the calculated maximum usable frequencies. To what extent the decrease due to this effect compensates for the increase due to the greater ratio of $f^{\prime} / f$ must be determined in individual cases.

Let us first consider the case of a linear gradient of ionization density, where $z=k f_{0}{ }^{2}$. Here $d z / d x=k f^{2}$ and the determination of $z_{v}$ and $z_{v}^{\prime}$ is easy. We obtain

$$
\begin{gathered}
z_{v}=3 z_{0}\left(\frac{1-a}{a}\right)\left[-1+\frac{1}{\sqrt{a(1-a)}} \tan ^{-1} \sqrt{\frac{a}{1-a}}\right] \\
z_{v}^{\prime}=z_{0}\left[1+\frac{1}{\sqrt{a \cos ^{2} \phi_{1}\left(1-a \cos ^{2} \phi_{1}\right)}} \tan ^{-1} \sqrt{\frac{a \cos ^{2} \phi_{1}}{1-a \cos ^{2} \phi_{1}}}\right] .
\end{gathered}
$$


Figure 1 shows the ratio of $z_{v}^{\prime} / z_{v}$ for $a=1 / 3$, which is the same as the ratio of $z_{v}{ }^{\prime}$ for $a=1 / 3$ to $z_{v}{ }^{\prime}$ for $a=0$, plotted against $\cos \phi_{1}$. It shows also the ratio $\left(f^{\prime} \cos \phi_{1} / f\right)^{2}$ for $a=1 / 3$, which is the same as the square of the ratio of $f$ for $a=0$ to $f$ for $a=1 / 3$, plotted against $\cos \phi_{1}$. The two ratios are equal at about $\cos \phi_{1}=0.8$. This means that, for the distance corresponding to this value of $\cos \phi_{1}$, the wave frequency corresponding to reflection at a point on the linear part of a $\left(z_{0}, f^{2}\right)$ curve is the same for $a=0$ as for $a=1 / 3$. At shorter distances the wave frequency is less, and at greater distances it is greater, for $a=1 / 3$ than for $a=0$.

If the $\left(z_{v}, f^{2}\right)$ curve curls up, as happens near a critical frequency, an increase in the height of the equivalent triangular path has relatively less effect than in the case of a linear $\left(z_{v}, f^{2}\right)$ curve. This means a relative increase in the wave frequencies calculated for points on a curve near a critical frequency. Thus it may be expected that the maximum usable frequencies calculated for an actual distribution of ionization density will be, for $a=1 / 3$, equal to or less than those calculated for $a=0$ only for very short distances. Elsewhere they will be greater, and may become as much as 20 percent or so greater, at the greater distances, than in the case where $a=0$. The calculation must in general be made for each individual distribution of ionization density.

The calculation can be made for a general distribution of ionization by assuming the $\left(z_{v}, f^{2}\right)$ curve to be made up of a number, $m$, of linear intervals. In the $n$th such interval the ionization density, represented by $f_{0}^{2}$, is assumed to vary between $f_{n}^{2}$ and $f_{n+1}{ }^{2}$, while the height varies from 0 to $z_{n}$. In this interval, then

$$
z\left(f_{0}{ }^{2}\right)=\frac{f_{0}{ }^{2}-f_{n-1}{ }^{2}}{f_{n}{ }^{2}-f_{n-1}{ }^{2}} z_{n} .
$$

Then, if $x=f_{0}{ }^{2} / f^{2}$ and $f_{m}{ }^{2}=f^{2}$,

$$
\frac{d z}{d x}=\frac{f^{2} z_{n}}{f_{n+1}{ }^{2}-f_{n}{ }^{2}}
$$

and is constant over the interval, so that eq 7 becomes

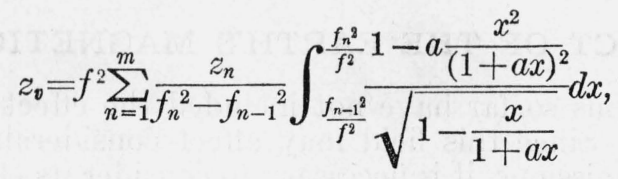

and eq 8 becomes

$$
z_{v}^{\prime}=f^{2} \sum_{n=1}^{m} \frac{z_{n}}{f_{n}^{2}-f_{n-1}^{2}} \int_{\frac{f_{n-1}^{2}}{f^{2}}}^{\frac{f_{n}^{2}}{f^{2}}} \sqrt{\frac{1+a \frac{(1-a) \cos ^{2} \phi_{1}}{1-a \cos ^{2} \phi_{1}}}{1-(1-a) x}} d x .
$$

Each of the integrals in the summation represents a part of the complete integral from 0 to $1 /(1-a)$ and may be readily evaluated for a given $a$ and $\cos \phi_{1}$. Both eq 11 and 12 are linear in $z_{n}$ and may easily be evaluated. If we know only the $\left(z_{v}, f\right)$ curve, eq 11 may be turned inside out and the known values of $z_{0}$ used to form $n$ equations in the $n$ unknown values of $z_{n}$, which can then be substituted in eq 12 to obtain $z_{0}^{\prime}$. 
Calculations of the maximum usable frequencies for $E$ - and $F_{2}$-layer transmission were made, using this method, for $a=0$ and $a=1 / 3$, for typical distributions of ionization density. The ratios of the maximum usable frequency for $a=1 / 3$ to that for $a=0$ are plotted, for various values of distance $D$, in figure 2 . The solid graph is for $F_{2}$-layer and the dotted for $E$-layer transmission.

For $F_{2}$-layer transmission, it is seen that the maximum usable frequencies are not much greater for $a=1 / 3$ than for $a=0$ until the distance of transmission exceeds about 500 kilometers. For greater

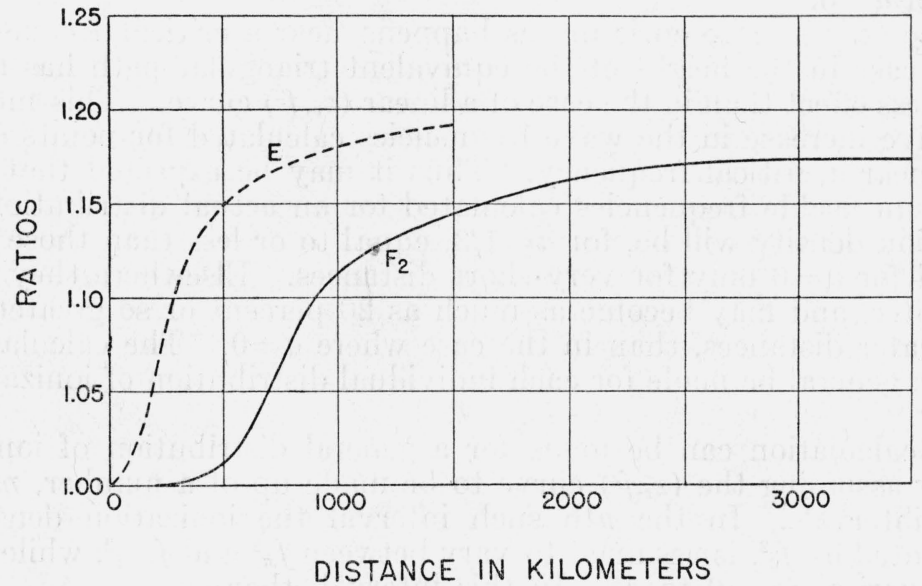

FigdRE 2.-Ratios of maximum usable frequencies for $a=1 / 3$ to those for $a=0$, for $E$ - and $F_{2}$-layer transmission.

distances the maximum usable frequencies for $a=1 / 3$ are higher than those for $a=0$, becoming about 17 percent higher at the greater distances.

For E-layer transmission the ratio of maximum usable frequency for $a=1 / 3$ to that for $a=0$ rises much more rapidly with distance than in the case of $F_{2}$-layer transmission, and attains a somewhat greater value.

\section{EFFECT OF THE EARTH'S MAGNETIC FIELD}

The calculations so far have not included the effect of the earth's magnetic field. Since this field may affect considerably some highfrequency transmissions, it is necessary to consider its effect. Accordingly calculations were made for the transmission path and frequency, for which experimental results are given below.

The transmission path was practically perpendicular to the magnetic meridian, and was about $650 \mathrm{~km}$ long. The earth's field was assumed to have an intensity of 0.5 gauss, and a dip of $70^{\circ}$. For a frequency of $6.06 \mathrm{Mc} / \mathrm{s}$, and a vertical-incidence virtual height of $130 \mathrm{~km}$ at the equivalent vertical-incidence frequency, table 1 gives the calculated oblique-incidence data. These were calculated on the basis of an average distribution of ionization density in the $E$ region, using the magneto-ionic equations as published by Booker [9]. 
TABLE 1.-Calculated oblique-incidence data

\begin{tabular}{|c|c|c|c|c|c|}
\hline $\begin{array}{c}\text { Earth's } \\
\text { field }\end{array}$ & $\begin{array}{c}\text { Value of } \\
a\end{array}$ & $\begin{array}{c}F_{o} \text { at } \\
\text { level of } \\
\text { of reflec- } \\
\text { tion }\end{array}$ & $\begin{array}{c}\text { Angle of } \\
\text { incidence }\end{array}$ & $\begin{array}{c}\text { Equivalent } \\
\text { vertical- } \\
\text { incidence } \\
\text { frequency }\end{array}$ & $\begin{array}{c}\text { Vertical- } \\
\text { incidence } \\
\text { critical } \\
\text { frequency }\end{array}$ \\
\hline Gauss & & $M c / s$ & Degrees & $M c / s$ & $M c / s$ \\
0 & 0 & 2.25 & 68.2 & 2.25 & 2.45 \\
0 & $1 / 3$ & 2.34 & 67.8 & 1.91 & 2.08 \\
0.5 & 0 & 2.20 & 68.7 & 2.20 & 2.40 \\
.5 & $1 / 3$ & 2.31 & 68.1 & 1.89 & 2.06 \\
\hline
\end{tabular}

The table gives, for $a=0$ and $1 / 3$ and for $H=0$ and 0.5 gauss, the ionization density at the level of reflection, the angle of incidence of the waves which traverse the given path, the equivalent vertical-incidence frequency, and the vertical-incidence critical frequency which corresponds to a maximum usable frequency of $6.06 \mathrm{Mc} / \mathrm{s}$ over the given path, via E-layer transmission. It may be seen that the inclusion of the earth's field where $a=0$ makes a difference of but 2.0 percent, and where $a=1 / 3$ a difference of but 1.0 percent. The inclusion of the polarization term, however, makes a difference of 15.1 percent where $H=0$ and 14.2 percent where $H=0.5$ gauss.

Thus it should prove easy to decide between the Lorentz and Sellmeyer theories by measuring the vertical-incidence $E$-layer critical frequency at the times when the oblique-incidence $E$-layer transmission begins and fails. The experimental error should be considerably less than the difference between the two theories, either for calculations based on $H=0$ or for closer calculations based on $H=0.5$ gauss. The calculations for east-west transmission may be made for $H=0$ with an error much less than the probable experimental error.

A recent analysis has been published by Ratcliffe [10] for a parabolic distribution of ionization density in the ionosphere. The curves and discussion he gave for oblique-incidence radio transmission indicated little difference between the results for $a=0$ and $a=1 / 3$, especially for long-distance transmission via the $F$ layer. Indeed, he stated that the difference would be greatest for a transmission distance of about $500 \mathrm{~km}$.

The curves of figure 2 make this statement appear in error. It seems likely that Ratcliffe was misled by making calculations only for ranges below about $700 \mathrm{~km}$, as published in his paper, for it is obvious that the greater separation between the two cases comes for considerably greater distances. It is possible, moreover, that the assumption of a parabolic distribution might also tend to lessen the difference at the shorter distances.

The results of the step-by-step analysis of typical $E$ - and $F$-layer curves indicate the nature of the difference in maximum usable frequency according to the two theories.

\section{EXPERIMENTAL OBSERVATIONS}

There is thus a clear distinction between the Lorentz theory $(a=1 / 3)$ and the Sellmeyer theory $(a=0)$ in the case of radio waves incident obliquely upon the ionosphere. It should therefore be possible to decide experimentally between the two theories. 
There are available considerable published data on oblique-incidence radio transmission $[11,12,13,14,15]$. Experiments involving the $E$ layer, which, as shown by figure 2, should provide conclusive results for relatively short transmission distances, are for the most part unreliable, usually because of the presence of sporadic- $E$ reflections above the true $E$-layer critical frequency. Such an experiment was attempted by Farmer and Ratcliffe [11], with somewhat inconclusive results. Experiments involving the $F_{2}$ layer have for the most part been conducted over distances of less than 600 kilometers. They are subject to the difficulty that ionosphere conditions at the point of reflection are much more uncertain for the $F$ layer than for the $E$ layer. Also, the difference between the two theories would produce a discrepancy of but a few percent, which is of the same order of magnitude as the uncertainty of ionosphere conditions at the point of reflection. Much of the published data on radio transmission, therefore, is of little use in deciding whether $a=0$ or $1 / 3$.

Since September 1935, the National Bureau of Standards has made continuous automatic field-intensity records of numerous radio stations. Results are available for the whole period from 1935 to 1940 for high-frequency international broadcasting station W8XAL (later WLWO), $6.06 \mathrm{Mc} / \mathrm{s}$, at Mason, Ohio, 645 kilometers distant. Some results of these recordings were presented in a paper before the joint meeting of the International Scientific Radio Union and the Institute of Radio Engineers at Washington in April 1936, and other results have been used by investigators at the Bureau in studies of the ionosphere $[16,17]$.

The transmissions from W8XAL to Washington were propagated normally by way of the $E$ layer during most of the daylight hours, and by way of the $F$ layer at other times. The transitions from $F$ - to $E$-layer transmission in the morning, and vice versa in the afternoon, were often well marked, since the vertical radiation pattern of the transmitting antenna was such as to provide substantially greater radiation for single-hop $E$ - than for $F$-layer transmission. The transitions have been identified on a great many records by the following facts. (1) The regularity of occurrence of the change in intensity and character of fading on the records corresponds to the regularity of the diurnal and seasonal variations of the $E$-layer critical frequency. (2) There was a complete absence of any regular sudden change in local ionosphere characteristics (critical frequency, virtual height, or absorption), except for the E-layer critical frequency, at the time of the change on the record. (3) The beginning of propagation was observed when no other ionosphere layer but the $E$ layer had sufficient ionization density to support transmission (as during the mornings of ionosphere-storm days). (4) The change on the record nearly always occurred about 15 minutes after the local $E$-layer critical frequency rose or fell through about $2.45 \mathrm{Mc} / \mathrm{s}$. This time difference is the difference between the local times at Washington and at the midpoint of the path from W8XAL to Washington.

Figure 3 shows four examples of the change of layer on the W8XAL records. The record of May 12, 1938, was made when a severe ionosphere storm had considerably depressed the critical frequencies of the $F_{1}$ and $F_{2}$ layers. At the time when good transmission began, none of the layers except the $E$ layer had even nearly enough ionization to support transmission. Records of February 21 and 22, 1938, were 

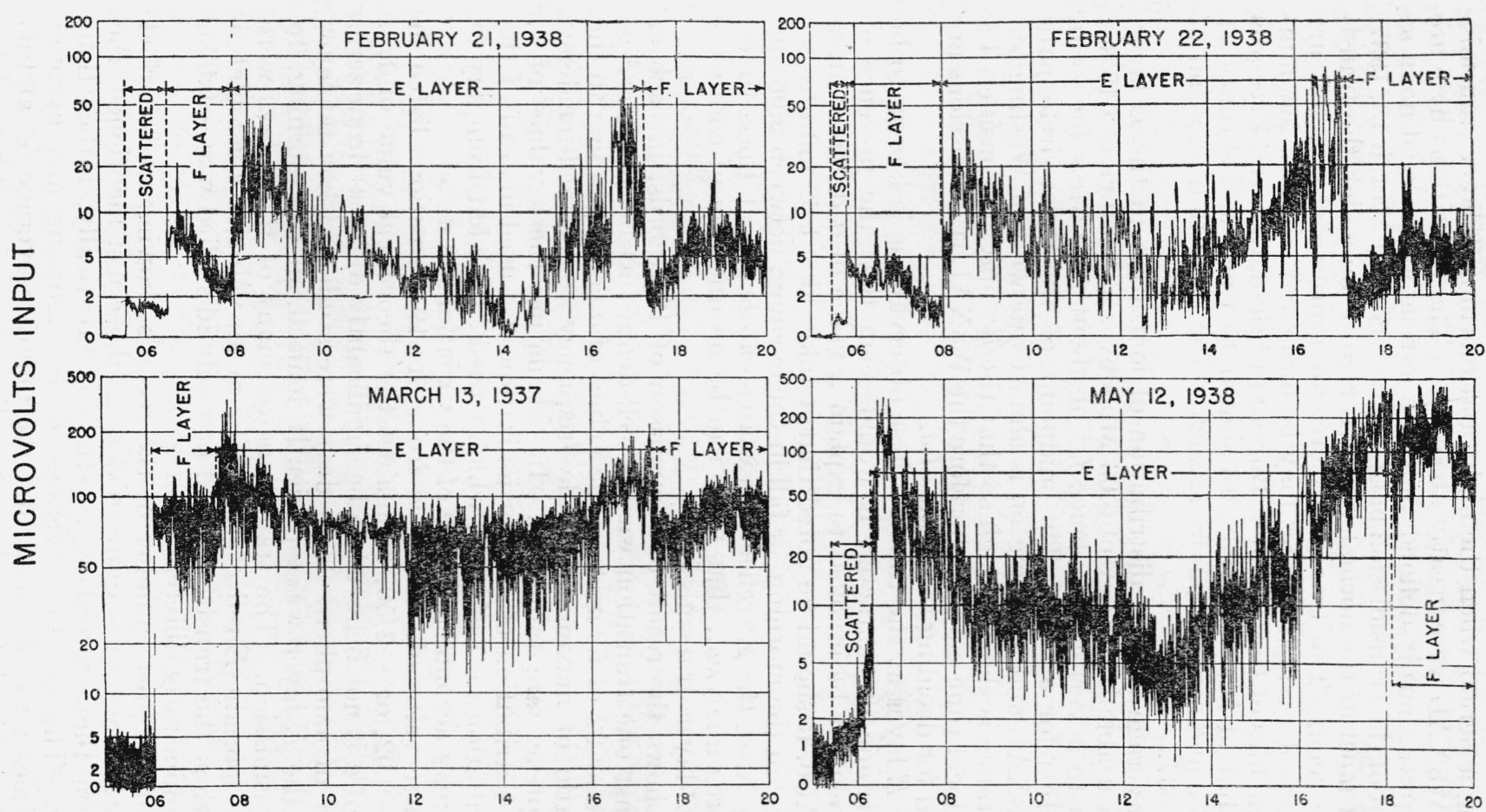

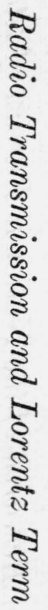

EASTERN STANDARD TIME

FIGURE 3.-Transitions between $E$ - and $F_{2}$-layer transmission from Mason, Ohio, to Washington, $D$. $C$. Station W8XAL, $6.06 \mathrm{Mc} / \mathrm{s}, 650 \mathrm{~km}$ distant. 
made during a period when the night and morning $F$-layer transmissions from W8XAL were greatly absorbed. The transitions here are seen to be quite sharply defined and symmetrical about local noon at the midpoint of the transmission path. The record of March 13, 1937, is typical of most of the normal W8XAL records on which the transition can be seen. The transitions are not quite so sharp, but are easily identifiable, and the times may be measured within a few minutes. In the files of the National Bureau of Standards are 11 records similar to that of May 12, 1938, 13 similar to those of February 21 and 22,1938 , and several hundred showing the transition like that of March 13, 1937.

For the average regular distribution of ionization in the $E$ layer, a maximum usable frequency of $6.06 \mathrm{Mc} / \mathrm{s}$ over a distance of $645 \mathrm{~km}$ corresponds to a vertical incidence $f_{E}{ }^{\circ}$ of about $2.40 \mathrm{Mc} / \mathrm{s}$ for $a=0$, and $2.10 \mathrm{Mc} / \mathrm{s}$ for $a=1 / 3$. The midpoint of the great-circle path between W8XAL and Washington is almost due west of Washington and the distance is short enough so that the ${f_{E}}^{\circ}$ may be considered a function of local time only. Therefore the W8XAL transmissions are well adapted for deciding the value of $a$.

Since the $E$ layer at the time of these observations is known to be chiefly electronic in character, it would seem that the presence of heavy ions cannot be invoked to explain a value of $a$ different from $1 / 3$ or 0 . Also, it should be noted that it takes about 32 minutes for the $f_{E}{ }^{\circ}$ to rise in the morning, or fall in the evening, between 2.06 and $2.40 \mathrm{Mc} / \mathrm{s}$. Also, the $E^{x}$ reflections were so absorbed at these times, on the records analyzed, that there can be no question of confusing $E^{\circ}$ - with $E^{x}$-layer transmission.

Table 2 shows the result of comparison of 13 records, in each of which at least one transition was as well-defined as in the record of February 21 or 22 of figure 3 . The values of $a$ corresponding to the observed ratio of maximum usable frequency to vertical-incidence critical frequency are tabulated. In calculating these values of $a$, an average distribution of $E$-layer ionization was used instead of the actual distribution for each day, which varied somewhat from day to day. This may account for some of the variations in $a$.

The average value of $a$ deduced from the 19 transitions listed in table 2 is -0.02 , or $-1 / 50$, as against the theoretical value of $1 / 3$. This value of $a$ is not believed to be significantly different from zero.

A number of ionosphere storm days were studied, when no layer other than the $E$ layer was sufficiently ionized, in the morning, to produce transmission. The transitions on many of these days were obscured by sporadic- $E$ reflections. The mean value of $a$ calculated on 7 days when the transition could be easily identified was -0.005 , again not significantly different from zero.

Because of the great number of the records showing well-marked transitions which were not quite so clear as those just mentioned, the transitions on these records were studied statistically, rather than individually. The monthly averages of the morning and evening transition times were obtained, and the monthly average $f_{E}{ }^{\circ}$ at this time was determined from the vertical-incidence ionosphere data. Table 3 shows the average values of $f_{E}{ }^{\circ}$ at the midpoint of the transmission path, at the times when E-layer transmission began and failed, and the corresponding values of $a$. The mean value of $a$, deduced from 435 transitions studied in this group, was -0.06 , 
again not significantly different from zero. The negative value of $a$ probably means that the height of the $E$ layer was slightly greater than that assumed as an average.

TABLE 2.-Values of " $a$ " calculated from value of local $f_{E}$ at time of change of layer for W8XAL, $6.06 \mathrm{Mc} / \mathrm{s}, 650 \mathrm{~km}$ distant

\begin{tabular}{|c|c|c|c|c|}
\hline \multirow{2}{*}{$\begin{array}{c}\text { Date } \\
\text { (February } \\
1938)\end{array}$} & \multicolumn{2}{|c|}{ Local $f_{B}$ in $\mathrm{Mc} / \mathrm{s}$} & \multicolumn{2}{|c|}{ Calculated value of $a$} \\
\hline & A. M. & P. M. & A. M. & P. M. \\
\hline $\begin{array}{r}6 \\
14 \\
15 \\
17 \\
18 \\
21 \\
22 \\
23 \\
24 \\
25 \\
26 \\
27 \\
28\end{array}$ & $\begin{array}{l}2.33 \\
2.48 \\
2.35 \\
2.44 \\
2.46 \\
2.48 \\
2.48 \\
2.54 \\
2.39 \\
2.36 \\
2.44\end{array}$ & \begin{tabular}{c}
2.47 \\
2.43 \\
-2.46 \\
2.45 \\
2.48 \\
\hdashline 2.37 \\
2.29 \\
2.35 \\
\end{tabular} & $\begin{array}{r}+0.10 \\
-.10 \\
+.08 \\
-.05 \\
-.07 \\
-.10 \\
-.10 \\
-.19 \\
+.02 \\
+.06 \\
-.05\end{array}$ & $\begin{array}{r}-0.08 \\
-.03 \\
-.07 \\
-.06 \\
-.10 \\
+.07 \\
+.15 \\
+.08\end{array}$ \\
\hline
\end{tabular}

TABLE 3.-Mean values of " $a$ " calculated from values of local $f_{E}$ at time of change of layer for $W 8 X \mathrm{AL}, 6.06 \mathrm{Mc} / \mathrm{s}, 650 \mathrm{~km}$ distant

\begin{tabular}{|c|c|c|c|c|c|c|}
\hline \multirow{2}{*}{ Month } & \multicolumn{3}{|c|}{ Morning transition } & \multicolumn{3}{|c|}{ Evening transition } \\
\hline & Time & $f_{E}^{\circ}$ & $a$ & Time & $f_{B}^{\circ}$ & $a$ \\
\hline 1936 & & & & & & \\
\hline $\begin{array}{l}\text { May.-- } \\
\text { June.-- }\end{array}$ & $-2-2$ & ... & ....- & $\begin{array}{l}1823 \\
1838\end{array}$ & $\begin{array}{l}2.50 \\
2.45\end{array}$ & $\begin{array}{r}-0.13 \\
-.06\end{array}$ \\
\hline July & & & & 1830 & $\begin{array}{l}2.40 \\
2.42\end{array}$ & $=.00$ \\
\hline September... & 0703 & 2.44 & -0.05 & 1737 & 2.40 & +.01 \\
\hline October & 0738 & 2.52 & -.16 & 1651 & 2.42 & -.02 \\
\hline November... & 0810 & 2.40 & +.01 & 1610 & 2.47 & -.08 \\
\hline December.... & 0831 & 2.48 & -.10 & 1559 & 2.53 & -.17 \\
\hline 1937 & & & & & & \\
\hline February.. & 0805 & 2.45 & -.06 & 1709 & 2.42 & -.02 \\
\hline March & 0726 & 2.47 & -.08 & 1739 & 2.40 & +.01 \\
\hline April & 0637 & 2.40 & +.01 & 1759 & 2.50 & -.13 \\
\hline May........... & 0604 & 2.42 & -.02 & 1818 & 2.48 & -.10 \\
\hline 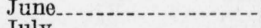 & -n-n- & $\cdots-\cdots$ & $\cdots$ & 1849 & 2.40 & +.01 \\
\hline July .- & & & & 1852 & 2.44 & -.05 \\
\hline Mean $a_{-}$ & & ... & -0.06 & -....... & -.......... & -0.06 \\
\hline
\end{tabular}

\section{CONCLUSIONS}

For the cases considered in this paper, the data indicate that the Lorentz polarization term should not be included in the theory of oblique-incidence radio transmission by way of the ionosphere. Further experiments in different latitudes and with careful determinations of oblique and vertical-incidence penetration frequencies should be performed before this conclusion can be positively confirmed.

It is suggested that experiments of this type, involving wide ranges of frequencies and reflection heights, are less conducive to misleading results and ambiguous interpretations than are vertical-incidence experiments in the neighborhood of one frequency, which involve somewhat uncertain factors, such as the magnitude and direction of the earth's magnetic field at definite heights, and assumptions as to the physical constitution of the ionosphere. 


\section{REFERENCES}

[1] H. A. Lorentz, Theory of Electrons (G. E. Stechert, New York, N. Y., 1909).

[2] C. G. Darwin, Proc. Roy. Soc. (London), [A] 146, 17 (1934).

[3] H. G. Booker and L. V. Berkner, Constitution of the ionosphere and the Lorentz polarization term, Nature 141, 562 (1938).

[4] D. F. Martyn and G. H. Munro, The Lorentz polarization term and the earth's magnetic field in the ionosphere, Nature 141, 159 (1938).

[5] E. V. Appleton, F. T. Farmer, and J. A. Ratcliffe, Magnetic double refraction of medium radio waves in the ionosphere, Nature 141, 409 (1938).

[6] N. Smith, Extension of normal-incidence ionosphere measurements to obliqueincidence radio transmission, J. Research NBS 19, 89 (1937) RP1013.

[7] N. Smith, Application of vertical-incidence ionosphere measurements to obliqueincidence radio transmission, J. Research NBS 20, 683 (1938) RP1100.

[8] D. R. Hartree, Proc. Cambridge Phil. Soc. 2\%, 143 (1930-31).

[9] H. G. Booker, The propagation of wave packets incident obliquely upon a stratified doubly refracting ionosphere, Roy. Soc. Phil. Trans. (London) $23 \% \mathbf{A}, 411$ (1938).

[10] J. A. Ratcliffe, The effect of the Lorentz polarization term in ionospheric calculations, Proc. Phys. Soc. (London) 51, 747 (1939).

[11] F. T. Farmer and J. A. Ratcliffe, Wireless waves reflected from the ionosphere at oblique incidence, Proc. Phys. Soc. (London) 48, 839 (1936).

[12] F. T. Farmer, C. B. Childs, and A. Cowie, Critical frequency measurements of wireless waves reflected obliquely from the ionosphere, Proc. Phys. Soc. (London) 50,767 (1938).

[13] E. V. Appleton and J. A. Ratcliffe, Proc. Roy. Soc. (London) 128, 133 (1930).

[14] D. F. Martyn, R. O. Cherry, and A. L. Green, Proc. Phys. Soc. (London) 4\%, 125 (1934).

[15] W. Crone, K. Kruger, G. Goubau, and J. Zenneck, Echo measurements over long distances, Hochfrequenztechnik Elektroakustik 48, 1 (1936).

[16] J. H. Dellinger, Sudden disturbances of the ionosphere, J. Research NBS 19, 111 (1937) RP1016.

[17] N. Smith and S. S. Kirby, Critical frequencies of low ionosphere layers, Phys. Rev. 51, 890 (1937).

Washington, October 28, 1940. 